\title{
Expert system for repeatability testing of high-performance liquid chromatographic methods
}

\author{
M. MULHOLLAND ${ }^{* \cdot a}$ and N. WALKER \\ Philips Scientific, Cambridge (UK) \\ F. MARIS and H. HINDRIKS \\ Organon International, Oss (Netherlands)
}

L. BUYDENS

University of Nijmegen, Nijmegen (Netherlands)

T. BLAFFERT

Philips Research Laboratories, Hamburg (Germany)

and

\section{P. J. SCHOENMAKERS}

Philips Research Laboratories, Eindhoven (Netherlands)

\begin{abstract}
A repeatability test is performed as part of the validation of the precision of a high-performance liquid chromatographic method. The purpose of the test is to establish the random errors of the method with respect to various method features. It is usual to test the repeatability of the sample preparation and the injection procedure by a number of repetitions. The number of repetitions depends on the application of the method. For instance, a quality control method is expected to analyse up to and over $\mathbf{5 0}$ samples in a single run. The repeatability test of the injection process therefore needs to reflect this number, in order to identify fully potential errors. These errors could be due to the instrument error or to drifting factors such as temperature. The expert system described provides the necessary expertise to identify the required tests and interpret the results. It also contains a diagnosis module which can identify sources of unacceptable errors. The diagnosis module is linked to a reoptimization process which can modify the method to improve the resolution between a critical pair of peaks. This expert system was built as part of the ESCA project, which is a 3-year project investigating the application of expert systems to analytical chemistry.

A repeatability test requires both heuristic and algorithmic knowledge and therefore provides a good challenge for expert system technology. The system is implemented in a multiple windows software environment and uses workstation hardware. This software has been evaluated in practical laboratory environments and some of the results and conclusions are described. The major advantage of an expert system implementation appears to be that it can give advice when problems occur. It also ensures that the statistics are used and interpreted correctly. This is not possible using conventional algorithmic software.
\end{abstract}

\section{INTRODUCTION}

High-performance liquid chromatography (HPLC) is usually applied as a quantitative technique, where the relative concentrations of components in a mixture

\footnotetext{
a Present address: University of New South Wales, Sydney, Australia.
} 
are determined. Each sample application requires a unique combination of chemical and instrumental conditions. The development of a method is a complex series of choices and optimizations which consider features of both the chemistry of the sample and its application. When developing a method, the analyst has certain expectations of its quantitative performance. These include accuracy, sensitivity, specificity and precision. To ascertain whether the new method achieves the expected levels of performance requires a series of validation experiments. Each validation test can be defined in four stages:

(1) The method characteristic is defined; this could be accuracy, precision or any other performance characteristic which is important to the application.

(2) The actual test is defined; for precision this could be repeatability, reproducibility of ruggedness.

(3) An experimental procedure with which to test these characteristics is then selected and carried out. This includes the measurement of various method characteristics.

(4) The final stage in a method validation test is to diagnose the results. This requires pass/fail criteria and a method for identifying and curing problems. It may sometimes require the reoptimization of the method if it fails to meet the specified performance levels.

HPLC method validation is thus an integral part of process of method development. Links back to method optimization are often required. It is also necessary to be aware of the performance requirements during the method development process. For instance, a method developed on a delicate microbore column would not be suitable for multiple usage throughout busy quality control laboratories.

This paper describes an expert system built to tackle these problems and create these links for a repeatability test. The system is built as part of a research project investigating the use of expert systems in analytical chemistry, ESCA.

A small expert system was initially built as a test case for the expert system development tool Goldworks. It used a combination of spreadsheets and expert system facilities such as frames and rules [1]. However, as the work of ESCA progressed it became clear that analysts did not just need stand-alone packages but communication links throughout the method development process. It was therefore decided to build a system which could perform a repeatability test and also communicate with the method optimization process. Three expert systems have been built which tackled the various stages in the method development process: first guess [2], selectivity optimization [3] and optimization of the instrumentation [4,5]. Links to the latter system looked most promising for the purpose of re-optimizing methods which had failed a method validation test. However, links or other systems would eventually be required in order to form a complete picture [6].

\section{STRUCTURE OF THE EXPERT SYSTEM}

The structure of the system is illustrated in Fig. 1. The system starts by consulting the system optimization module. This module optimizes the physical parameters such as flow-rate, column dimensions and detector flow cell. The aim of the optimization is to provide the fastest analysis time within the required resolution. In the stand-alone system the optimization limits consist of the hard physical limits on the 


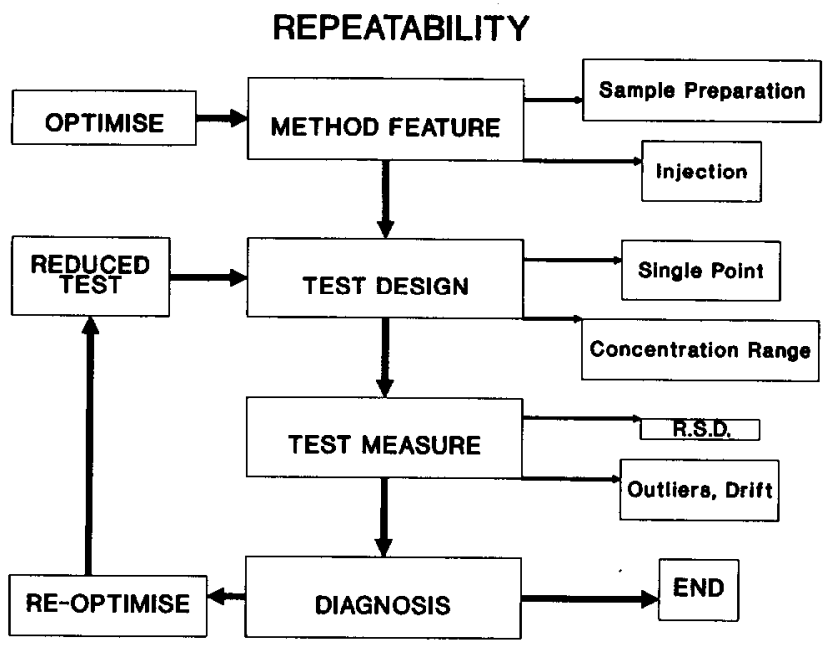

Fig. 1. Overview of the repeatability test expert system.

instrument capability, the available sample volume, with user-defined ranges for the resolution and signal-to-noise ratio of the analysis. The role of these limits changes when they are applied to optimizing a method which has predefined constraints on its repeatability. The limits need to specify ranges within which an optimized method should be repeatable. Some of these limits are shown in Table I. They are divided into three categories: the possible range, the reliable range and the robust rangc. The range is selected by examining the method application. Some examples are shown in Table I. When the required range is specified the system optimizes for the fastest analysis time. The results include the following information: (1) the selected column dimensions; (2) the selected detector. flow cell; (3) the value of the time constant; (4) the flow-rate and pressure drop of the instrument; (5) the projected analysis time, resolution and signal-to-noise ratio for the analysis; and (6) the selected values for the

TABLE I

RANGE REQUIREMENTS FOR THE OPTIMIZATION

\begin{tabular}{llll}
\hline Parameter & Possible range & Reliable range & Robust range $^{a}$ \\
\hline Flow-rate & $0.01-10 \mathrm{ml} / \mathrm{min}$ & $0.1-5 \mathrm{ml} / \mathrm{min}$ & $0.3-3 \mathrm{ml} / \mathrm{min}$ \\
Pressure & $0-400 \mathrm{bar}$ & $5-250 \mathrm{bar}$ & $5-200 \mathrm{bar}$ \\
Resolution & 1 & 2 & 3 \\
Signal-to-noise ratio & 10 & 100 & 200 \\
Column I.D. & $0.25-25 \mathrm{~mm}$ & $2-8 \mathrm{~mm}$ & $4-8 \mathrm{~mm}$ \\
Examples: & $<25$ & 25 & 25 \\
$\quad$ Sample No. & 1 & $>1$ & $>2$ \\
\hline Lab. No. & & & \\
\hline
\end{tabular}

a The range of values within which the method is likely to be robust, i.e., not affected much by small changes. 
TABLE II

AN EXAMPLE OF A PEAK TABLE

\begin{tabular}{llcllll}
\hline $\begin{array}{l}\text { Peak } \\
\text { No. }\end{array}$ & $\begin{array}{l}\text { Retention time } \\
(\mathrm{s})\end{array}$ & $\begin{array}{l}\text { Peak height } \\
(\mathrm{mm})\end{array}$ & Peak area & Asymmetry & Plate count & $\begin{array}{l}\text { Relevant: } \\
\text { yes/no }\end{array}$ \\
\hline 0 & 79 & 20 & 1000 & 1 & 23000 & yes \\
1 & 91 & 70 & 1000 & 1 & 23900 & yes \\
2 & 145 & 100 & 1000 & 1 & 21700 & yes \\
3 & 180 & 20 & 1000 & 1 & 24900 & yes \\
\hline
\end{tabular}

sample concentration and injection volume. The user can interact with these results by selecting from several options. When one is satisfied with the optimization, the repeatability test can begin.

The method features to be tested are then defined as the sample preparation and the injection procedure. The analyst inputs a description of the HPLC method together with information on the expected usage. With this information an experimental design can be recommended. Originally, the system recommended a number of repetitions of the sample preparation and the injection procedure at a single concentration point. However, during the evaluation this was found to be inadequate. Therefore, designs were added to deal with multiple concentration levels.

After the design has been recommended, the user carries out the experiments and collects data for retention times, peak areas, peak heights and concentration. These data are input to the system using peak tables; an example is shown in Table II. The user is only allowed to proceed to the next stage if all the peak tables are entered and all have the same number of peaks.

The test measure is now made on these data. For each peak, the relative standard deviation (R.S.D.) of each value is calculated [1]. These measurements are then used to diagnose the repeatability of the method.

The diagnosis is performed in several stages:

(1) The concentration variations are measured against a pass/fail criterion, usually $1 \%$. If they pass then the diagnosis ends here and the method is concluded to be repeatable. However, if it fails it then proceeds to the next stage.

(2) A Grubbs test for outliers is performed and the user is allowed to remove any outliers identified.

(3) The results can be viewed graphically to determine any drifting conditions.

(4) Potential problems are diagnosed and a cure is suggested.

TABLE III

CLASSIFICATION OF R.S.D. VALUES

\begin{tabular}{llll}
\hline Classification & Height R.S.D. (\%) & Area R.S.D. (\%) & Retention time R.S.D. (\%) \\
\hline Small & 0 & 0 & 0 \\
Medium & 1 & 1 & 0.5 \\
Large & 2 & 2 & 1 \\
\hline
\end{tabular}


The R.S.D. values are used to identify possible problems. The R.S.D.s are first classified as small, medium or large according to Table III. The combined behaviour of these values can indicate specific problems. For instance, if multiple injections of the same sample gives rise to large variations in peak height and area, but not in the retention times, then this could be due to a variation in injection volume or possibly degradation of the sample. The diagnosis section of the repeatability test gives a list of possible problems with the method. It does this in four stages.

(1) The classifications of small, medium and large are used to create lists of possible problems for each peak. Each problem within a list has a priority or weight. A value of zero means that the problem is not possible. Two lists are formed, one for sample preparation problems and the other for injection problems.

(2) The individual lists of problems are combined into two overall lists by summing the priorities of each problem over the peaks.

(3) The list of problems are then checked. Certain problems, although diagnosed, can be discounted if the chromatographic method contains the correct features. For instance, if $\mathrm{pH}$ variation is diagnosed but the method is utilizing a buffer then this problem can be discounted.

(4) The list of problems with injection and sample preparation are combined into one complete ordered list. The list of problems with injection is considered to be more important than those for sample preparation, so are given a higher priority. If a problem occurs in both lists then the higher of the two priorites is taken. Once the problem list has been established the system goes on to recommend corrective actions.

Each possible problem diagnosed has a list of actions that can be taken to try to solve the problem. These actions can be divided into two categories: actions that involve changing the method, such as the re-optimization of resolution, and actions that involve checking or maintaining the chromatograph. The expert system provides suggested actions to the user based on their priority score and checking and maintenance cures are always performed first. If a certain action does not solve the problem, this is measured by performing a reduced test, then the next highest priority is suggested. If the method cannot perform after any recommended checking or maintenance cures then some reoptimization is suggested. If the resolution between a pair of peaks falls below a predefined level throughout repeatability test, then the user is routed to the system optimization module. This causes the resolution to be increased to a larger value. Fig. 2 shows a graphical representation of the diagnosis process.

The software is implemented using Pascal in a multiple windows environment. A fuller description of the software and hardware environment is given elsewhere.

This system was evaluated using several pharmaceutical examples. The results for the analysis of ethinylestradiol are presented here.

\section{EXPERIMENTAL}

\section{Sample preparation}

Samples are prepared by adding a formulated tablet containing ethinylestradiol to $2.0 \mathrm{ml}$ of an internal standard solution of estradiol. This is then shaken and sonicated until the tablet is completely dispersed. The mixture is centrifuged and $10 \mu \mathrm{l}$ of the supernatant are injected onto the column. 


\section{TEST MEASURE}

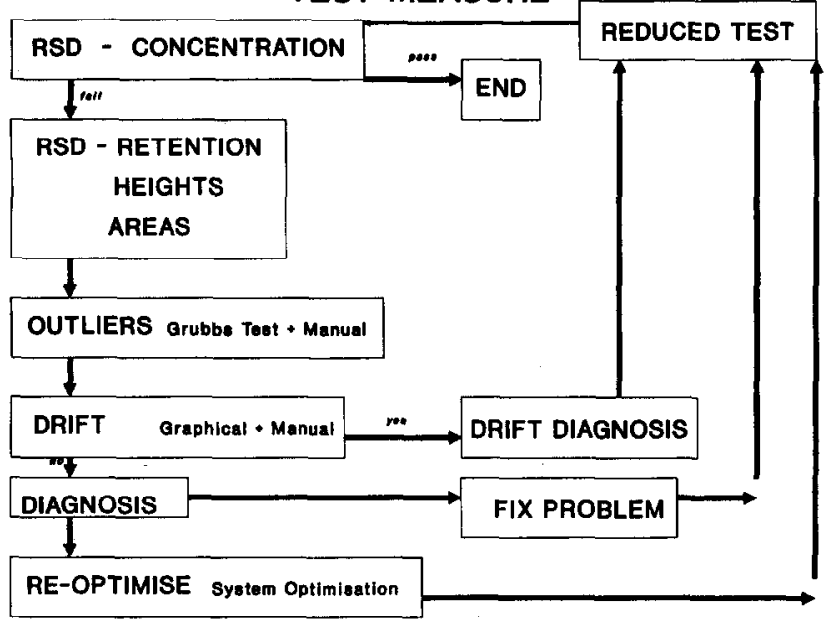

Fig. 2. Overview of the measurement and diagnosis modules.

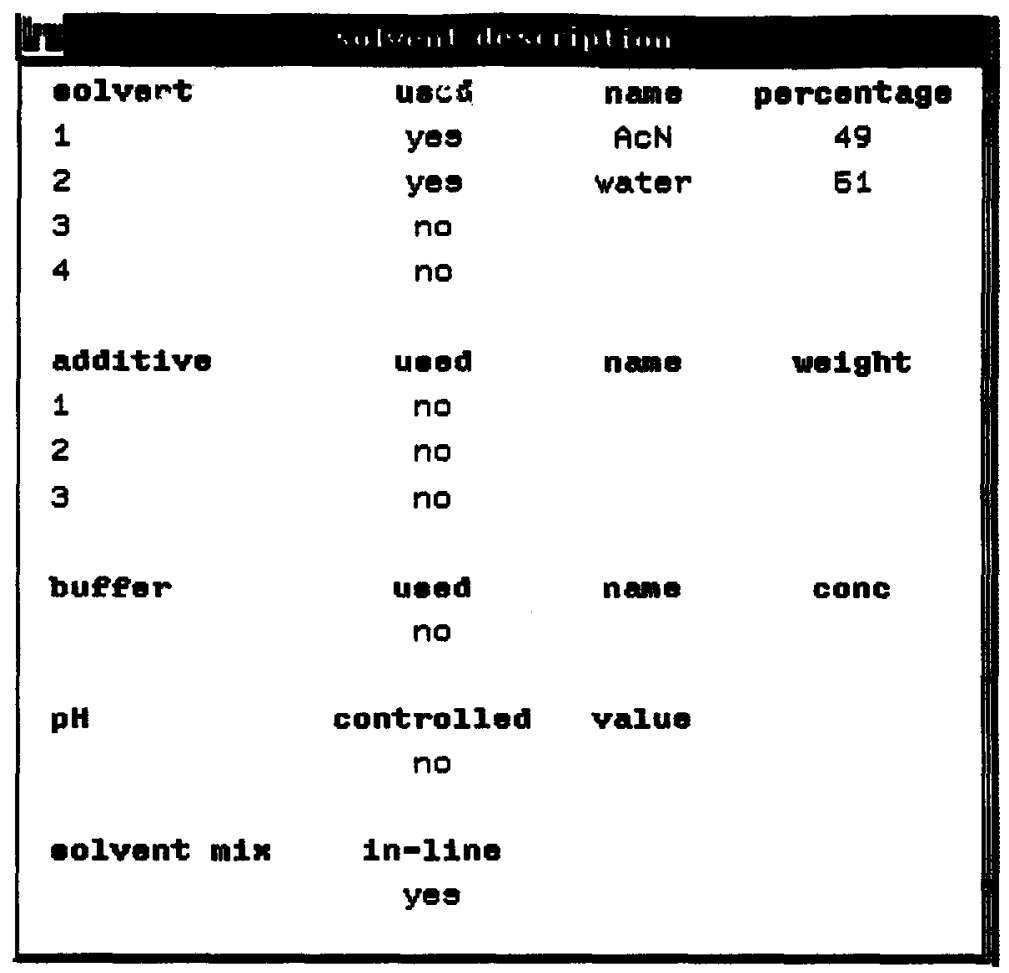

Fig. 3. Chromatogram of ethinylestradiol (latest eluting peak) with estradiol as the internal standard. 


\section{Chromatographic conditions}

The sample is eluted from a $100 \times 5 \mathrm{~mm}$ I.D. Novapak $\mathrm{C}_{18}$ column with a mobile phase of acetonitrile-methanol-phosphoric acid (60:40:0.05) at a flow-rate of $2 \mathrm{ml} / \mathrm{min}$. The column temperature is ambient and the sample is detecting using a UV detector at $205 \mathrm{~nm}$.

\section{RESULTS}

Fig. 3 shows as an example a chromatogram of ethinylestradiol together with estradiol. This method is input to the system using a number of tables reserved for this information, an example of the solvent description is shown in Fig. 4. The recommended experimental design involves a total of 98 injections. This includes the injection of standards to calibrate the instrument.

Fig. 5 shows the conclusions obtained from the diagnosis of these results. The calculated values for the R.S.D. are shown for each of the sample preparation tests and the injection procedure test. For the injection procedure test no problems are found and all the R.S.D.s have values below the medium classification shown in Table III. However, for the sample preparation a small problem is observed in the variation of peak heights and areas for peak 2 . The problem is diagnosed as due to either sample degradation or inadequate sample preparation. For this example the sample degradation is considered the priority problem. The suggested action is to

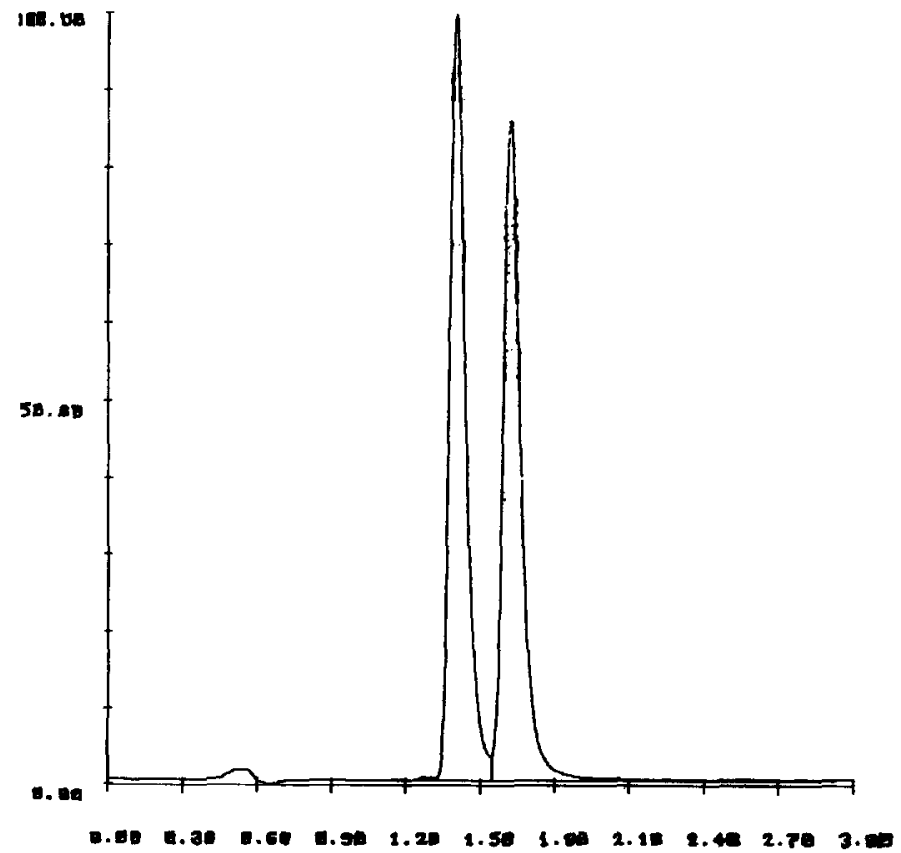

RETENTION TIME (MINUTES)

Fig. 4. Example input window for the solvent description. 

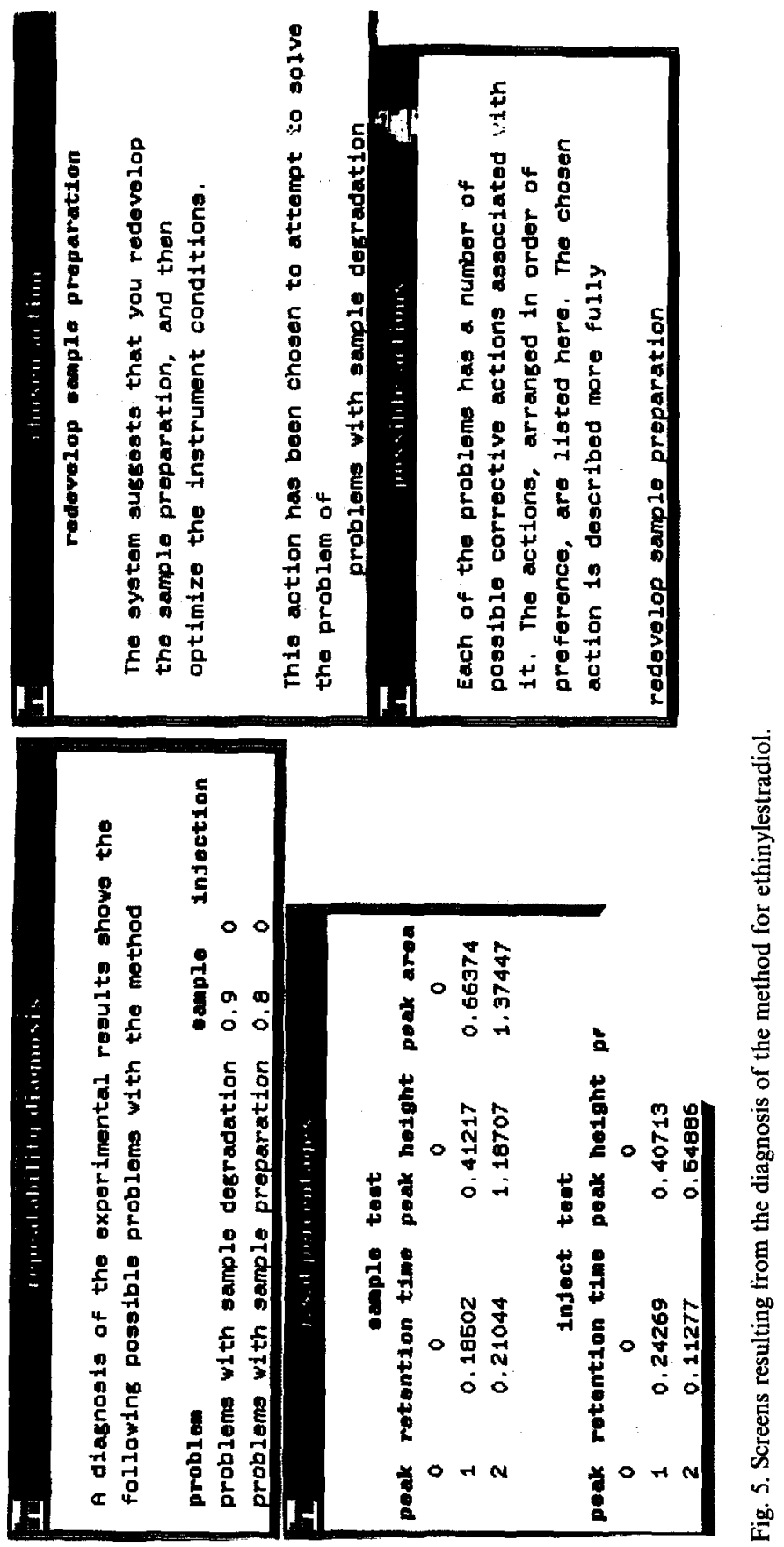


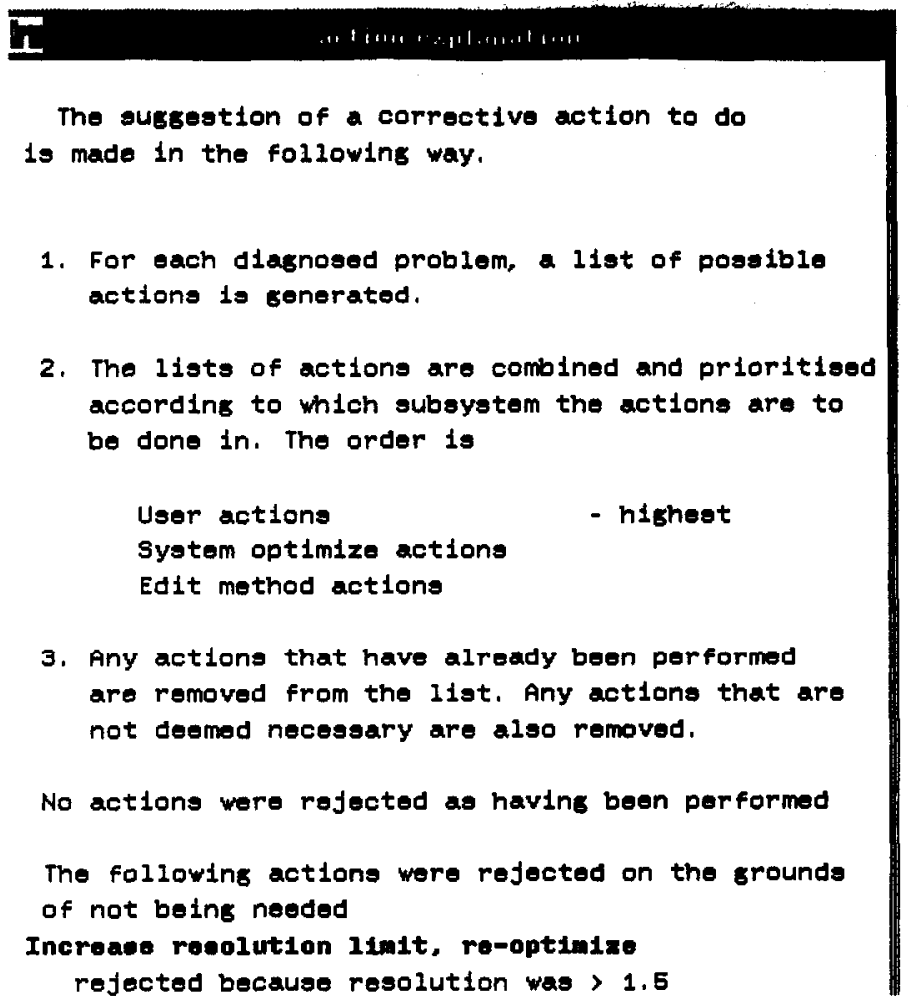

Fig. 6. Explanation provided for the suggested corrective actions.

re-develop the sample preparation. Fig. 6 shows the explanation available for this corrective action.

It is interesting that the evaluators felt that the measured performance is acceptable for this method, but were pleased to find the system suggesting actions that could further improve the method. This is a typical advantage of expert systems over conventional software packages.

\section{CONCLUSIONS}

The use of expert system technology to develop a repeatability test package is considered successful. The approach allows the use of both algorithmic and heuristic knowledge. Algorithmic knowledge, such as that required for R.S.D. calculation, can be implemented in a conventional equation, whereas heuristic knowlegde, such as that required for problem diagnosis, can be implemented as rules.

The evaluation of this system proved extremely valuable as it resulted in several additions which enhanced the software considerably. The software was shown to give good advice and even suggested improvements to methods that were previously considered acceptable.

However, several additions could still be made to complete the repeatability test 
system. It could be integrated with a chromatography data station to improve the efficiency of data transfer within the software. To complete the picture the system would need to be linked with the remainder of the method development process. This would allow any problems identified to be cured by re-developing the method.

\section{REFERENCES}

I M. Mulholland, J. A. van Leeuwen and B. G. M. Vandeginste, Anal. Chim. Acta, 223 (1989) 183-192.

2 H. Hindriks, F. Maris, J. Vink, A. Peeters, M. de Smet, D. L. Massart and L. Buydens, J. Chromatogr., 485 (1989) 255-265.

3 A. Peeters, L. Buydens, D. L. Massart and P. J. Schoenmakers, Chromatographia, 26 (1988) 101-109.

4 P. J. Schoenmakers, N. Dunand, A. Clelland, G. Musch and T. Blaffert, Chromatographia, 26 (1988) 37-44.

5 P. J. Schoenmakers and N. Dunand, J. Chromatogr., 485 (1989) 219-236.

6 P. Conti, H. Pityns, N. Vanden Driessche, M. de Smet, T. Hamoir, F. Maris, H. Hindriks, P. J. Schoenmakers and D. L. Massart, Chromatographia, submitted for publication. 